\title{
Local and Global Skeleton Fitting Techniques for Optical Motion Capture
}

\author{
Marius-Calin SILAGHI, Ralf PLÄNKERS, Ronan BOULIC, Pascal FUA and \\ Daniel THALMANN \\ Computer Graphics Lab, Swiss Federal Institute of Technology, CH-1015 Lausanne \\ Switzerland \\ E-mail: silaghi@lia.di.epfl.ch, \{plaenker, boulic, fua, thalmann\}@lig.di.epfl.ch
}

\begin{abstract}
Identifying a precise anatomic skeleton is important in order to ensure high quality motion capture. In this paper we discuss two skeleton fitting techniques based on 3D optical marker data. First a local technique is proposed based on relative marker trajectories. Then it is compared to a global optimization of a skeleton model. Various proposals are made to handle the skin deformation problem. Index Terms-skeleton fitting, motion capture, optical markers
\end{abstract}

\section{Introduction}

As stressed in a recent production featuring many virtual humans, the most critical element in their creation seems to be the replication of believable motion [1]. In most productions optical motion capture is preferred due to its high precision measurement of little reflective markers attached on some relevant body landmarks (Fig. 1a). The movement of an artist is captured with two to eight calibrated cameras. For simple motions the multiple views of markers allow the automatic reconstruction of their $3 \mathrm{D}$ position. Once per session, a special calibration motion that highlights all the necessary degrees of mobility allows to build or adjust a skeleton model (this motion is further referred to as the gym motion). Then the skeleton model is used in a postprocessing phase to derive the angular trajectories of all the captured motions. Finally animators often adjust angular data to adapt the motion to a virtual character that is different from the artist (Fig. 1d).

Presently, the stage of automatic 3D reconstruction is often brought to a halt for complex motions. Either some markers are obscured from camera view or the algorithm confuses the trajectory of one marker with that of another. This requires much manual intervention that severely reduces the productivity of the system. In the framework of the MOCA ESPRIT project, we propose a motion capture methodology based on an anatomic human model (Fig. 1b) [2]. This model encompasses a precise anatomic description of the skeleton mobility [3] associated with an approximated envelope. It has a double objective: by ensuring a high precision mechanical model for the performer, we can predict accurately the 3D location and the visibility of markers, thus reducing significantly the human intervention during the conversion 
process. In the present paper we discuss methods exploiting the gym motion to estimate the dimensions of the artist's skeleton.

Input motion

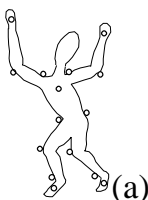

Human performer wearing optical markers (little reflective spheres)
MOCA framework<smiles>CCCC(CC)(CC)CC</smiles>

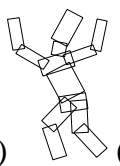

(c)

Anatomic Envelope for skeleton visibility assessment
Output motion

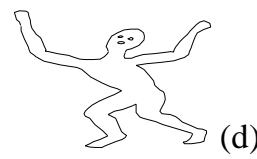

End user virtual character

Fig. 1. Converting the human performer's motion (a) into the end-user character's motion (d) with the anatomic human body (skeleton (b) and approximated envelope (c))

In the next section we review skeleton identification approaches used in other motion capture techniques and in related fields. Then we propose a local skeleton fitting technique based on the relative marker trajectories. In the fourth section we develop a global fitting that adjusts simultaneously all the parameters of the skeleton. We conclude by reviewing our results and by making a comparison between the two proposed techniques.

\section{Skeleton Fitting Techniques in Related Fields}

Besides optical markers systems, other techniques exist that are based on magnetic trackers [5][9] or plain video sequences [4][6][8].

The main advantage of the magnetic trackers lies in the possible real-time acquisition and the unambiguous measurement of tracked points. However, its main drawback comes from a lower precision that is even worse when the magnetic field is perturbed by metallic objects or other magnetic fields. Regarding skeleton identification, in [5] it reduces to the scaling of a standard model to the size of the real human. Other important differences are estimated by manual measurements and reflected on the skeleton model.

The video-based motion capture techniques try to fit a human model to video sequences. Monocular or stereo video sequences may be used. The monocular case uses a planar projection [6] of a human body model. The parameters of the projection can model global scaling. The technique belongs to the image processing family of techniques and therefore the quality of the recording is strongly sensitive to noise. Another problem in this case is an undetermined direction. These drawbacks disappear when using stereo sequences. In [4] an arm recorded with a stereo system is being tracked by fitting a model built out of ellipsoids to the data. This way, the skeleton fitting is concomitant to the motion tracking. The complex body parts where the deformation of muscles has to be modeled as well, introduces a number of parameters that is proportional to the number of ellipsoids. The measures of the body 
are modeled by parameters of the ellipsoids and they are globally adjusted over all or selected frames of the sequence.

Other fields also rely on a precise identification of human features. In order to create virtual mannequins that behave exactly like the real human model for presenting garments, the body of the client has to be measured acceptably well [7]. To perform such measurements, two digital cameras capture frontal and side silhouette of customers. Although such an approach is efficient to build a suitable 3D envelope of the client, it fails to identify precisely the joint location [7].

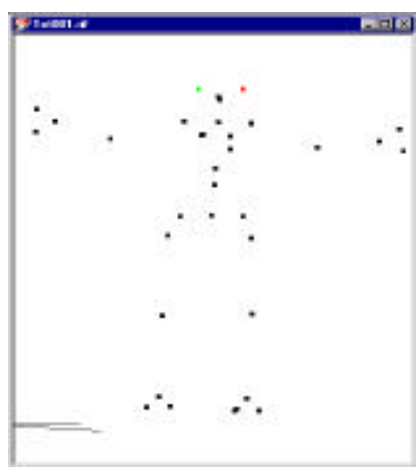

Fig. 2. A frame containing 3D optical markers from a gym sequence, used as input for the skeleton fitting

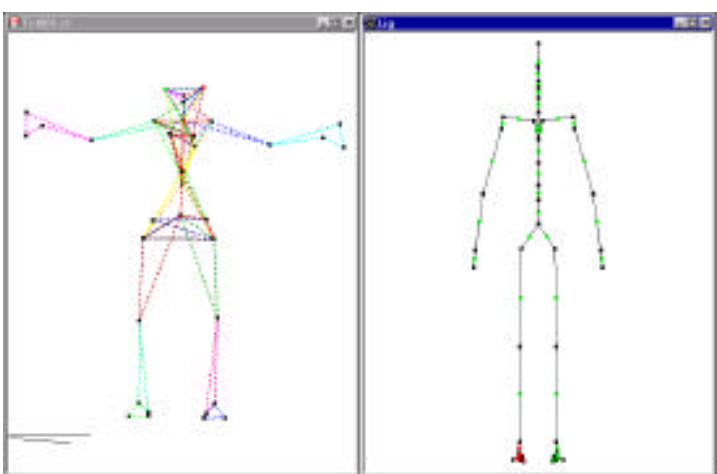

Fig. 3. Interface for correction and association of markers' sets with skeleton segments. Result of tuning the threshold (left). Skeleton model template (right)

The use of optical markers (Fig. 2) simplifies the human model without loss of precision, increasing significantly the speed of the computations while avoiding all the aforementioned problems. A problem lies in the possibility of loosing occluded markers during complex motions. This is the point where the rigorous fitting of an anatomic skeleton proves to be especially important. Using it, accurate prediction of the artist's posture can be made and integrated in the marker identification procedure. It supports suitable decisions in discarding hidden markers out of the alternatives of important choices at the $2 \mathrm{D}$ level of a camera view.

\section{Local Technique}

When looking for the position of the bones of a person, a first observation is that the relative distance of markers attached to one limb is almost constant. The biggest deviations occur when markers are attached on parts that suffer maximal deformation during the movement as around the joints or on massive muscles (e.g. on the thigh). Our approach handles this context by decomposing the problem into three stages developed in the following subsections:

- Partitioning the markers into rigid segment sets

- Estimating the position of joints

- Deriving the corresponding skeleton dimensions 


\subsection{Partitioning}

We have to specify which marker belongs to which segment. This can be done manually by using an anatomic skeleton and making associations. Nevertheless, an automatic tool is welcomed (Fig. 3). We propose an algorithm that computes the distances between markers at each frame of the gym motion (Fig. 4). It selects the biggest sets of markers in which all distance variations between all pairs of markers are under a certain threshold. This condition defines a rigid segment set. The system may look for the expected number of partitions or the user can interactively tune this threshold (Fig. 3).

We call partition such a rigid segment set. Its formal definition is the following: we define a relationship $R$ over the set of markers. The relationship $R$ determines a cover $C$ over the set of markers $M$.

$$
C=\left\{P \mid P \subset M, \forall m_{1}, m_{2} \in P, m_{1} \neq m_{2} \Rightarrow R\left(m_{1}, m_{2}\right)\right\}
$$

where

$$
R\left(m_{1}, m_{2}\right) \equiv\left(m_{1}, m_{2}\right) \in R
$$

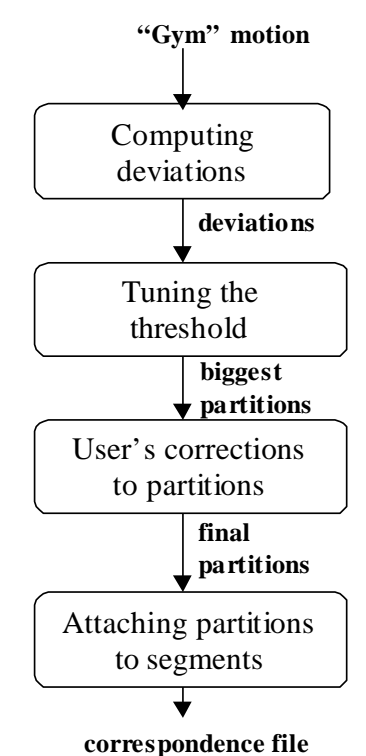

Fig. 4. Steps followed in partitioning

Now we define the cover $\mathfrak{R}$ over $M$ as:

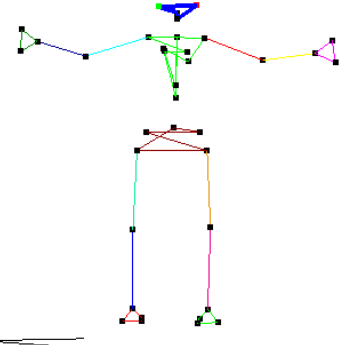

Fig. 5. Maximal Partitions after corrections and association with segments of the template

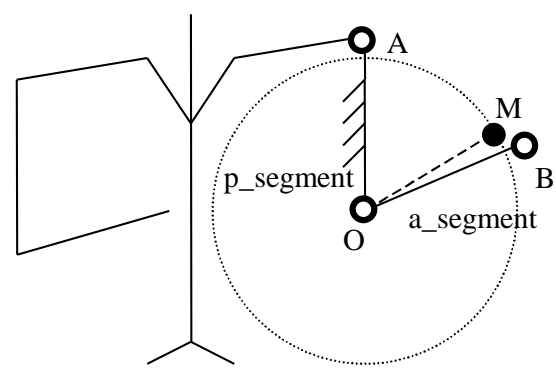

Fig. 6. The trajectory of a marker $M$ around an adjacent segment $\mathrm{OA}$ 


$$
\Re=\{P \mid P \in C, \forall Q \in C, Q \neq P \Rightarrow P \not \subset Q\}
$$

We call $\mathfrak{R}$ "the set of biggest partitions of markers found in the relationship $R$ ".

In our case two markers $m_{1}$ and $m_{2}$ are in the relation $R$ if, over all frames, the difference between the longest and shortest distances $d\left(m_{1}, m_{2}\right)$ between the markers, passes below a threshold $\theta$. Thus $R$ is the set:

$$
\left\{\left(m_{1}, m_{2}\right) \mid \max _{\text {frames }} d\left(m_{1}, m_{2}\right)-\min _{\text {frames }} d\left(m_{1}, m_{2}\right)<\theta\right\}
$$

We intend to further develop this tool using information based on the angles formed by the markers. Corrections can be done manually. After computing the sets in $\mathfrak{R}$ we need to establish a relationship between each of such set (Fig. 5) and a segment of the skeleton model. In the current stage we are doing this association manually, using an interactive graphical interface (Fig. 3). We will try AI learning techniques based on the motion of the local systems of coordinates to accomplish it (see section 3.3).

We define the attachment weight of a marker to a segment as a normalized measure of the rigidity of its attachment to that segment. By default, all the attachment weights have a value of 1.0.

\subsection{Using the Relative Trajectories of the Markers}

If we consider a referential bound to a bone represented by a segment e.g. OA (Fig. 6), the markers that are attached on adjacent segments (e.g. OB) theoretically move on a sphere centered on the joint that links the two segments (here joint $O$ ). This comes from the hypothesis of constant distance between markers and joints.

The position of a segment in space is completely defined by three points. Thus, if we have a minimum of three markers on a segment, we can define the position and orientation of that segment in space. Afterwards, we compute the movement of the markers on adjacent segments in the referential established by these markers and we estimate their centers of rotation (Fig. 10). The centers of rotations correspond to the joints. From their position in space we can compute the lengths of the segments as the distances between them. For example, in Fig. 6 we can compute the position of the joints $\mathrm{A}$ and $\mathrm{O}$ in space and we get the distance $\|\mathrm{AO}\|$.

Due to the deformations suffered by the skin during the motion, the markers attached on a limb change their position with respect to the bone. As long as the deformation is only due to a twisting rotation, it is filtered out by its property of maintaining the distance to the joints. However, a deformation that is changing the distance to the bone (e.g. due to muscles such as biceps) or one that changes the position along the bone induces unknown errors. Markers suffering such deformation errors are further said to belong to the noisy class. We deal with these errors by introducing a LSQ computation of the center of rotation. We use a modified version of the LevenbergMarquardt method [10] for all of our least squares computations. Depending on the complexity of the movements, the errors sum up or compensate each other, the worst cases being presented in [12]. 


\subsection{Segment Referential}

Due to skin deformations, during motion the markers may change their position with respect to the underlying bones. This induces errors in computing a referential bound to a segment. The biggest errors result from the displacement that may affect the origin of the system of coordinates. In order to filter out such errors we assign little weights to the markers that belong to the noisy class, and we choose the origin as the center of mass of the weighted markers. In order to improve the stability of the direction of the axis, we first choose the farthest marker from the origin for the direction of one axis. In our case we compute this way the Ox axis. This marker is then marked during the whole video sequence. Then we define the plan $\mathrm{xOy}$ as the plan that contains the marker situated at the biggest distance from the axis Ox. All the distances used in comparisons are multiplied with the attachment weight because the increase in reliability is proportional to the distance.

The choice of the two important markers that determine the referential is done only once. The calibration frame is the first frame of the sequence in which all the markers attached to the reference segment ( $p \_s e g m e n t$ in the formula) are visible. They are used for computing the referential in all the frames. The user manually specifies the weights. We provide an interactive display that allows checking the effect of the weights (Fig. 11 and Fig. 12).

\subsection{Center of Rotation}

In the p_segment referential we compute all the centers of rotation for all the markers of an adjacent segment $a \_s e g m e n t$ (Fig. 6). The center of rotation is estimated as the result of the function:

$$
\underset{r, x_{0}, y_{0}, z_{0}}{\arg \min } \sum_{\text {trajectory }}(d(r, x, y, z) \times \text { weight }(r, x, y, z))^{2}
$$

corresponding to the LSQ minimization [10] of the function:

$$
d(r, x, y, z) \times \text { weight }(r, x, y, z)
$$

where:

$$
d(r, x, y, z)=\sqrt{\left(x-x_{0}\right)^{2}+\left(y-y_{0}\right)^{2}+\left(z-z_{0}\right)^{2}}-r
$$

and the function weight $(r, x, y, z)$ is described in the section 3.6.

Then we estimate the joint position as the center of mass of the centers of rotation weighted by the associated marker weight and the radius of the sphere that they describe (Fig. 7).

$$
\text { weight }_{\text {center }}=\frac{\text { weight }\left(\text { mrk, } a_{-} \text {segment }\right)}{\operatorname{radius}\left(m r k, p_{-} \text {segment }\right)}
$$


We have conjectured that the precision of the center estimation is related to the radius of the rotation and empirically we have used the previous formula. However, the relation between precision and radius proved, as shown in [12], not to be very tight.

Take as an example Fig. 7. After defining the system of coordinates bound to $S_{1}$, we estimate the center of rotation $\mathrm{J}$ of $S_{2}$ in this referential. In order to do this we estimate the center of rotation $\bar{x}_{J}$ of each of the markers $\mathrm{M}, \mathrm{N}$ and $\mathrm{P}$. Then we compute the mass center of the centers of rotation for $\mathrm{M}, \mathrm{N}$ and $\mathrm{P}$ using the weights computed with the previous formula:

$$
\bar{x}_{J}=\frac{\sum_{\text {centers }}\left(\bar{x}_{\text {center }} \times \text { weight }_{\text {center }}\right)}{\sum_{\text {centers }} \text { weight } t_{\text {center }}}
$$

There is a case where the trajectory of a marker describes a circle and not a sphere, due to reduced degree of freedom for a certain joint (e.g. elbow). We would project this trajectory in the plan that best contains it. This plan can be found by using a LSQ that minimizes the distance between it and the points on the trajectory (Fig. 9).

A certain attention has to be paid to the case where we have less than three attached markers on a segment. This case occurs often in our experiments (Fig. 12). Currently we solve it with two markers if the adjacent joints can be acceptably modeled as having only one rotational degree of freedom. In this case we determine the system of coordinates by the plane that contains the two markers of the base segment and the marker whose trajectory is being tracked. The center of rotation is computed in this plane and then retransferred into the global referential. There, we compute the center of mass of all the centers of rotation computed for all the markers on a neighbor segment in order to find an estimate for the position of the joint.

Afterwards, we perform as usually. For example (Fig. 6) we compute all the rotation centers of the markers on $\mathrm{OA}$ around $\mathrm{OB}$, and all the rotation centers of the markers on $\mathrm{OB}$ around OA. Then we compute the center of mass using the weights of the considered markers and the inverse of the radius of the circles or spheres described by them during their motion.

\subsection{Getting the Resulting Skeleton}

By applying the previously described procedures we get finally a set of joints estimates for each frame. The next step is to compute the length of each bone in the anatomical skeleton so that the previously computed joints can be in the estimated position in each frame.

One trivial approach is to estimate the length as the average distance between the estimated joints. A more elaborated one is to compute the length that minimizes the square of deviations. 


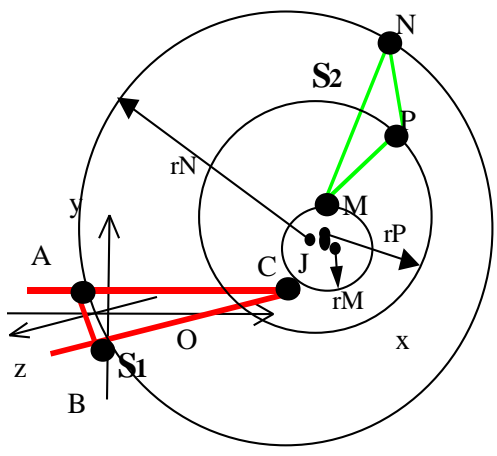

Fig. 7. Weighting centers of rotation for different markers on segment $S_{2}$

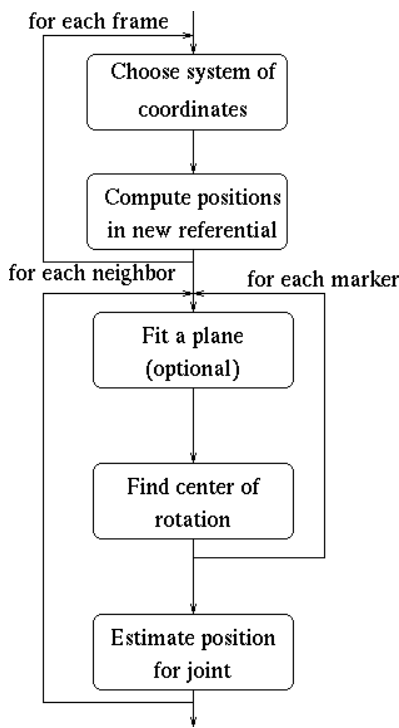

Fig. 9. Algorithm used to estimate the position of the joints for all the frames of the gym motion

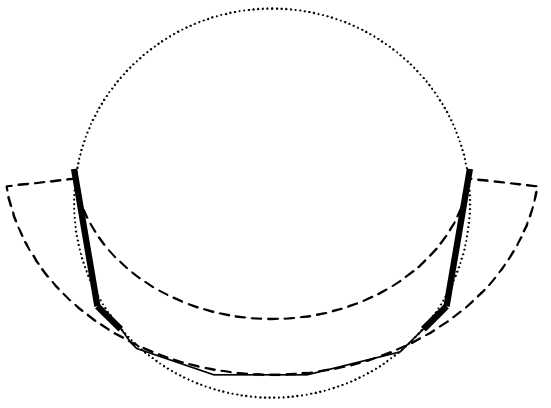

Fig. 8. Case where the lack of proper weighting would induce a bigger error than the ones described in the presented theory. The thickness of the continuous lines represents the density of the points in the trajectory

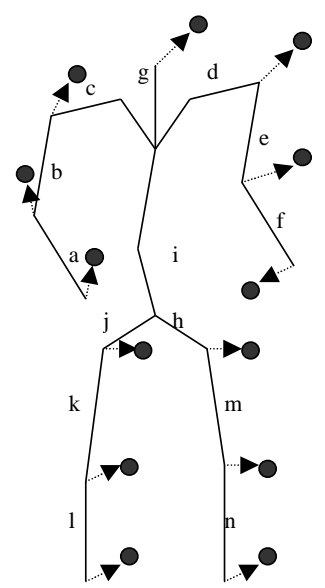

Fig. 10. Each segment length is multiplied with a coefficient (one of "a" to " $n$ ") and the degrees of freedom are modified in order to bring the model skeleton in the estimated position in each frame, marked here by the black circles

Currently we use a global adjustment of the lengths that minimizes the distance between the joints of a model and the estimated joints in each frame, adjusting in the same step all the other degrees of freedom of the model (Fig. 10). The same technique as the one presented in section 4.2 is employed.

We provide also the possibility of constraining the estimation of a symmetrical skeleton. This constraint can be set very easily in the context of using the global LSQ procedure mentioned above. The only change that has to be done in computations is 
to use the same coefficients for the adjustment of the symmetric lengths. For example, in Fig. 10 we would have:

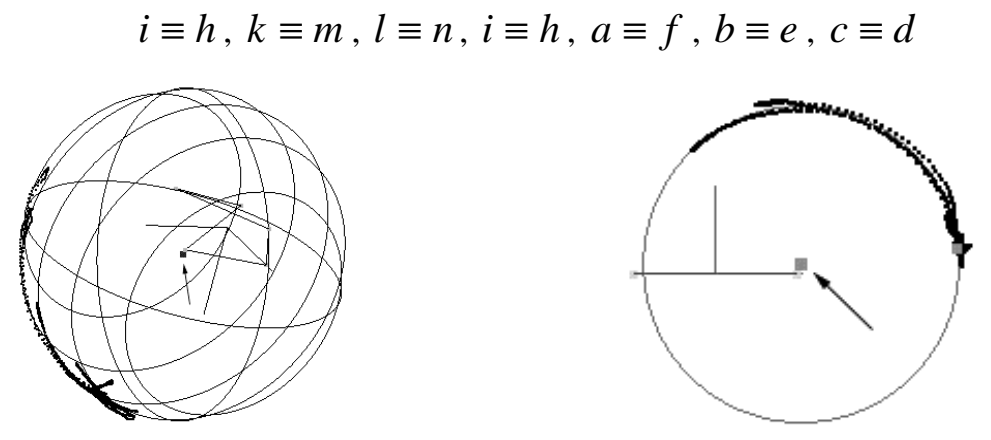

Fig. 11. Estimating the center of rotation of a marker around a segment determined by several
Fig. 12. Right upper arm. Example of a case where the segment can be determined by only 2 markers

\subsection{Errors, Advantages and Drawbacks}

In order to restrict the errors of the previously shown type we weight the points on the trajectories. The weight is the inverse of the densities of these points in a region of the space. We compute this densities by first dividing the space in a set of parallelepipeds in which we count the number of points. First we compute automatically the minimal box containing all the points of the trajectory and we divide it, dividing each direction by a factor of 5 or 10 . This increases the importance of poorly populated regions of the space representing extremities of the values of the degrees of freedom. Usually, the artist keeps for very short time such postures. Such an inverse density weight diminishes strong noise as the one in the Fig. 8.

As it is proved in 12 , if

$$
\varepsilon<R,
$$

where $\mathrm{R}$ is the shortest possible distance between the joint and the marker while $\varepsilon$ is the maximum deviation of this distance, then we can approximate the error $\mathrm{x}$ :

$$
x \cong \frac{\varepsilon}{1-\cos \left(\frac{\alpha}{2}\right)} \geq \frac{\varepsilon}{2}
$$

The last formula shows the maximum precision of the estimation of the position of a joint in a given direction. It is a function of the maximum angle $\alpha$ of the arc described by the marker in a plane determined by the joint and that direction vector. The maximum precision is less than half of the maximum deviation of the distance between marker and joint. 


\section{Global Technique}

We now present a different approach to fitting a skeleton to motion capture data. It is called global skeleton fitting and here it is used to refine the results of the local technique of section 3. Global means, that we consider the whole skeleton at once, whereas the local approach fits one limb at a time, ignoring relations between remote limbs. A thorough description of the global method can be found in [4].

Since this method depends heavily on the underlying skeleton structure, we first give a brief description of the used skeleton.

\subsection{Modeling the Skeleton}

As mentioned in the introduction, the skeleton of a body model is defined as a set of articulations connected by rigid segments [3]. The model's topology is defined by a hierarchy tree and may be of humanoid, animal or any other shape which allows for tree-like representation of the connected articulations. A "template" defines the translations and rotations that have to be affected to get from an articulation to its parent articulation in the hierarchy. This template describes the initial state of the skeleton. It may vary between different instances of a model, i.e. different characters or actors, but it is fixed for each instance during animation. Here, the local transformation matrix of a joint is multiplied by a "motion matrix", i.e. the matrix of the rotation around this particular joint which represents the model's motion.

Thus, the state of the skeleton is described by the state vector $P_{\text {body }}=\left[P_{\text {skel }}, P_{\text {motion }}\right]$.

The initial state of the skeleton $P_{\text {skel }}$ consists of the rotations and translations from each joint to its parent. It is fixed for a given body model. The variable state vector $P_{\text {motion }}$ contains the actual values for each DOF. They reflect the position of the body with respect to its rest position.

For any given limb or body part a partial state vector for its parent joint can be written as $P_{\text {part }}=\left\lfloor P_{\text {pre }}, Q_{i}\right\rfloor$, where $P_{\text {pre }}$ is the state vector of the preceding joint in the hierarchy, and $Q_{i}$ is the rotation angle of this DOF.

The position of joints in a global or world referential is obtained by multiplying the local coordinates with a transformation matrix. This matrix is computed recursively by multiplying all the transformation matrices that correspond to the preceding joints in the body hierarchy:

$$
X_{w}=\prod_{i} D_{i}(P) \times X_{l}
$$

with $X_{l, w}$ being local, resp. world, coordinates and the transformation matrices $D_{i}$, which depend on the state vector $P$, ranging from the root articulation's first to the reference articulation's last joint. 
The articulations may consist of several joints, each having its own transformation matrix $D=D_{r o t} \times D_{i n i}$. Take as example the elbow which has the two DOFs flex and twist: $D_{\text {elbow }}=D_{\text {rot }}^{\text {twist }} \times D_{\text {ini }}^{\text {twist }} \times D_{\text {rot }}^{\text {flex }} \times D_{\text {ini }}^{\text {flex }}$. The "initial transformation" $D_{i n i}^{k}=R \times X+p T, k=\{$ flex, twist,... $\}$ is a matrix directly taken from the LIG skeleton. It translates by the bone length and rotates the local coordinate system from this joint to its parent. The matrix entries are calculated with the values of the state vector $P_{s k e l}$. The variable coefficient $p \in P_{\text {skel }}$ is necessary because we don't know the exact size of the person's limbs yet. For the first joint of an articulation this matrix is usually dense but the other joints have no translation and the rotational part usually consists only of a permutation of the axes to ensure that the DOF rotates around the local z-axis.

\subsection{Fitting the Skeleton to Observations in a Global Approach}

Optical motion capture delivers accurate 3D positions of markers in world coordinates. We fit a model to these markers by minimizing a certain error function, which penalizes the distance between real marker position and the position predicted by the model. The employed model is depicted by Fig. 14. In order to be able to measure a distance, both "marker observations" need to be in the same referential. Thus, we transform the local coordinates of the modeled marker positions into world coordinates by multiplying them with the recursive transformation matrix $D(p)$.

The fitting is then done by minimizing the error $\varepsilon$ over all frames and all DOFs in the following equation:

$$
\sum\left\|O b s-X_{w}\right\|^{2}-\varepsilon=0
$$

where $X_{w}$ depends on the state vector $P$, as explained in the previous paragraph. To minimize the error, we employ the same LSQ algorithm as in section 3.2.

In other words, the global fitting searches optimal values for the DOFs and the lengths of the limbs by taking all frames into account.

\subsection{Practical Constraints versus Modeling Real Deformations}

Usually, the markers are modeled as being rigidly attached to the skin. But in reality they are effected by skin deformations. Those deformations are depicted by Fig. 13. We see that, due to the muscles (e.g. biceps), skin deformation at the joints and twisting, the markers are free to move in all directions. Allowing all those deformations in our model, even with limiting constraints, would lead to overparameterization. However we may consider that a certain position is the normal one while the others are unstable and less probable.

In this case, the fact itself that we use a LSQ technique models such deformations. Besides, some deformations are easily avoided by not attaching markers in noisy 
places. Usually markers are not attached on biceps or similar places so that the corresponding degree of freedom is sufficiently well modeled by the LSQ method. The deformation of the skin along the bones is important only on the joints. Several characteristics such as the attachment weight of markers (section 3.1) can be used. The deformations of the skin in transversal directions on the bones, due to twisting, are sometimes important. A limit may still be set on the extent of the deformation from one frame to another.

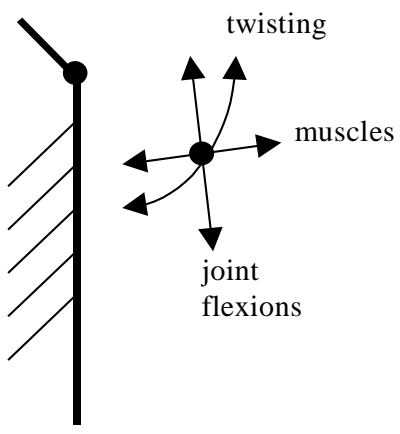

Fig. 13. Degrees of freedom and their cause for the movement of the markers with respect to segments

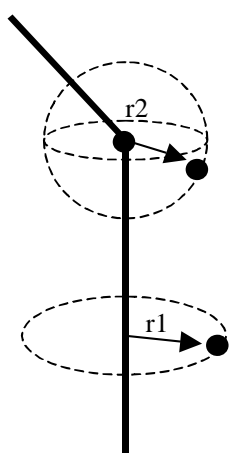

Fig. 14. The model of the markers on the segments

\subsection{Computational Issues}

The biggest drawback of the LSQ approach is that it gets stuck in local minima. The best solution to this problem is to ensure a good initialization. The random initialization is therefore avoided.

Taking into account the flexibility of the global approach regarding the models for the attachment of the markers on the limbs, we find it interesting to use the result of the local technique as the initialization of the global one. This way, as long as the model for the global LSQ is enough constraining, we are sure of improving the previously found result. The only condition is that the model should be more constraining than the local one, respectively the movement of the markers on circles around segments and weighted with attachment weights at joint proximity. The twisting model can be improved, eliminating the errors that come in the local technique from the computation of the referential.

\subsection{Considered Models, Errors, Advantages and Drawbacks}

A model of the markers on the limbs being currently considered is presented in the Fig. 14. The markers that are secured to only one segment are modeled as moving on a circle around the segment. The exact position as well as the radius of the circle is computed as a result of the LSQ optimization (section 4.2) in which these parameters are global over all frames. This approach allows the modeling of the markers attached 
at different distance on the same bone.

The markers secured very close to joints are modeled as moving on a sphere around the joint. The radii of these spheres are also globally optimized. We intend to research the effects of additional constraints regarding the parallel and meridian of the sphere on which the marker can be found as a function of the current values of the degrees of freedom.

Additional frame to frame constraints may be added in order to improve the convergence of the LSQ technique while taking into account the position of the marker on the corresponding circle or sphere.

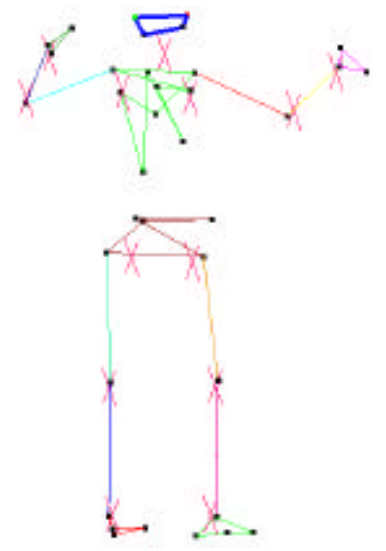

Fig. 15. Crosses show the position of some joints estimated using the local fitting technique

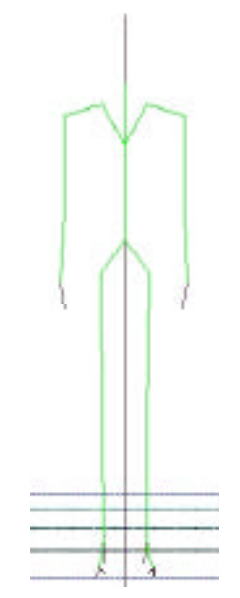

Fig. 16. Skeleton obtained by using a global adjustment of an anatomic skeleton to the positions of the joints estimated using the local algorithm

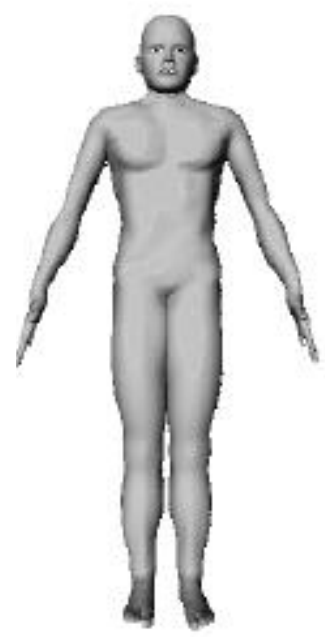

Fig. 17. Body obtained by covering the obtained skeleton with muscles and skin

\section{$5 \quad$ Results and Experiments}

We have tested the previously described algorithms on some "gym" motions. In Fig. 15 we present the computed positions in space for the joints using the technique presented in section 3. Out of these positions estimated in all the frames of the sequence we obtain the skeleton in Fig. 16 by applying the algorithm of section 3.5.

In addition to this skeleton we compute estimates of the position of the markers on the limbs. This estimation can be used in further improving the marker tracking and the analysis of the motion. After covering the computed skeleton with muscles and skin we obtain the skeleton of Fig. 17. 


\section{Local versus Global Approaches}

Until now we have encountered some problems with the convergence of the global LSQ. The main reason is that we have not used all the frames for the global technique, and because of this, the possibility of introducing frame to frame constraints was also reduced. Another reason is that the conversion of the results of the local technique into the needed parameters for the initialization of the global LSQ was done using yet another LSQ. We intend to further do this analytically. One promising direction is to set multiple priorities levels [11] instead of looking for one global compromise in the posture adjustment phase.

Two complementary validation campaigns are also planned. First recording optical marker data on an articulated structure with known dimensions (e.g. a robot). Second to exploit a set of simple human motions isolating various degrees of mobility in complex regions as the shoulder. The analysis of these data should lead to a better understanding of the markers optimal positioning.

A comparison between the two approaches is summarized in the next table:

\begin{tabular}{|l|l|}
\hline Local & Global \\
\hline Complex algorithm & Simple algorithm \\
\hline $\begin{array}{l}\text { Low computational cost } \\
\text { (high speed) }\end{array}$ & $\begin{array}{l}\text { High computational cost } \\
\text { (slow speed) }\end{array}$ \\
\hline $\begin{array}{l}\text { The manually specified weights are } \\
\text { important }\end{array}$ & $\begin{array}{l}\text { The importance of the values of the weights } \\
\text { depends on the employed model. }\end{array}$ \\
\hline $\begin{array}{l}\text { The model of the markers on the } \\
\text { limbs cannot be modified easily. }\end{array}$ & $\begin{array}{l}\text { The model of the markers on the body is } \\
\text { flexible. }\end{array}$ \\
\hline $\begin{array}{l}\text { Robustness within the limits of the } \\
\text { quality of the input }\end{array}$ & May easily get stuck in local minima \\
\hline
\end{tabular}

\section{Conclusion}

We have proposed a new approach to fitting a skeleton to motion capture data. It is based on two different, yet complementary techniques: a local and a global one. The local technique consists of the analysis of the relative trajectories of the markers. It proves to be very fast but good results are dependent on the quality of the gym motion. Also, its output is a set of independent 3D positions of the joints which still have to be assimilated to a skeleton. The global technique employs expensive computations for the simultaneous estimation of all parameters. In order to succeed it needs an already close initialization and a good constraining model, otherwise it gets stuck in local minima. However, it is less sensitive to incomplete motions and can better handle skin deformation. Its output is a complete articulated skeleton structure, ready to be used for animation purposes.

The combination of both techniques eliminates the problems each of them has on its own: the local technique serves as initialization to the global one, which delivers the fitted skeleton. 


\section{Acknowledgements}

We thank our MOCA partner, ACTISYSTEM, for their help in providing test data files as well as our colleagues in LIG for their technical help. The MOCA project is sponsored by the European ESPRIT program. This work is also partly supported by the Swiss Federal Institute of Technology, Lausanne.

\section{References}

1. Cinefex 72, Titanic special reprint, 98 pp, P.O. Box 20027, Riverside, CA 92516, USA, December 97

2. R. Boulic, P. Fua, L. Herda, M. Silaghi, J.-S. Monzani, L. Nedel and D. Thalmann "An Anatomic Human Body for Motion Capture” to appear in Proceedings of EMMSEC '98, Bordeaux

3. R. Boulic, L. Nedel, W. Maurel and T. Molet “Anatomic BODY Model, Kinematics", ESPRIT Project 25513 MOCA, D. Thalmann EPFL Project Director, 22 pages, December 1997

4. P. Fua, A. Grün, R. Plänkers, N. D'Apuzzo and D. Thalmann "Human Body Modeling and Motion Analysis From Video Sequences" International Symposium on Real Time Imaging and Dynamic Analysis, June 2--5, 1998, Hakodate, Japan.

5. Molet T., Boulic R., Thalmann D. "A Real-Time Anatomical Converter for Human Motion Capture" 7th EUROGRAPHICS Int. Workshop on Computer Animation and Simulation'96, Poitier, France, G. Hegron and R. Boulic eds., ISBN 3-211-828-850, Springer-Verlag Wien, pp 79-94.

6. S.Wachter, H.-H. Nagel "Tracking of persons in Monocular Image Sequences" Proceedings, IEEE Nonrigid and Articulated Motion Workshop, June 16,1997, San Juan, Puerto Rico.

7. Stephen Gray "Virtual Fashion" IEEE Spectrum, Feb. 98.

8. J.K. Aggarwal and Q. Cai "Human Motion Analysis: A Review" Proceedings, IEEE Nonrigid and Articulated Motion Workshop, June 16,1997, San Juan, Puerto Rico.

9. B. Bodenheimer, C. Rose, S. Rosenthal, J. Pella "The Process of Motion Capture: Dealing with data" 8th EUROGRAPHICS Int. Workshop on Computer Animation and Simulation'96, Poitier, Hungary, D. Thalmann and M. van de Panne eds., ISBN 3-21183048-0, Springer-Verlag Wien, pp 3-18.

10. W.H. Press, B.P. Flannery, S.A. Teukolsky, and W.T. Vetterling "Numerical Recipices, the Art of Scientific Computing" Cambridge U. Press, Cambridge, MA, 1986.

11. P.Baerlocher, R.Boulic "Task-Priority Formulations for the Kinematic Control of Highly Redundant Articulated Structures" To appear in Proceedings of IROS '98, Victoria Oct. 98

12. M.-C.Silaghi, R.Plànkers, R.Boulic, P.Fua and D.Thalmann"Local and Global Skeleton Fitting Techniques for Optical Motion Capture” Technical Report, LIG, EPFL, Sept. 98 